\title{
Adefovir-induced Fanconi syndrome associated with osteomalacia
}

\author{
Samel Park', Woo-ll Kim', Dai-Hyun Cho', Yeo-Joo Kim', Hong-Soo Kim', Ji-Hee Kim², Seung-Kuy Cha², Kyu-Sang Park², \\ Ji-Hye Lee ${ }^{3}$, Sang Mi Lee ${ }^{4}$, and Eun Young Lee ${ }^{1}$ \\ 'Department of Internal Medicine, Soonchunhyang University Cheonan Hospital, Cheonan; ${ }^{2}$ Department of Physiology, Yonsei University \\ Wonju College of Medicine, Wonju; ${ }^{3}$ Department of Pathology, ${ }^{4}$ Department of Nuclear Medicine, Soonchunhyang University Cheonan \\ Hospital, Cheonan, Korea
}

Fanconi syndrome is a dysfunction of the proximal renal tubules that results in impaired reabsorption and increased urinary loss of phosphate and other solutes. The pathophysiology of drug-induced Fanconi syndrome is unclear. Here we report the case of a 36-year-old woman who presented with pain in multiple bones and proteinuria. She had a 7-year history of taking adefovir at $10 \mathrm{mg} /$ day for chronic hepatitis B. Three years previously she had received surgery for a nontraumatic right femur neck fracture, after which she continued to complain of pain in multiple bones, and proteinuria, glycosuria, and phosphaturia were noted. The findings of a light-microscope examination of a renal biopsy sample were normal, but mitochondrial damage of the proximal tubules was evident in electron microscopy. Western blot analysis revealed that the level of serum fibroblast growth factor 23 (FGF23) was lower than in normal controls. After 2 months of treatment, hypophosphatemia and proximal tubular dysfunction were reversed, and serum FGF23 had normalized. This case suggests that direct mitochondrial damage in proximal tubules can cause drug-induced Fanconi syndrome associated with osteomalacia. (Clin Mol Hepatol 2018;24:339-344)

Keywords: Adefovir; Fanconi syndrome; Mitochondria; Proximal tubules; Osteomalacia

\section{INTRODUCTION}

Adefovir has been widely used for the treatment of hepatitis B and human immunodeficiency virus (HIV) infections. ${ }^{1,2}$ Several side effects associated with adefovir are reported, including acute renal failure and Fanconi syndrome (FS). ${ }^{3,4}$ FS results from dysfunction of reabsorption in the proximal tubules of the kidney. This impairment causes increased excretion of solutes, such as amino acids, glucose, uric acid, bicarbonate, and phosphate. ${ }^{5}$ Phospha- turia, in particular, leads to osteomalacia which results in symptoms such as muscle weakness, fatigue, bone pain, and fractures. ${ }^{6}$

The mechanism of drug-induced FS is unclear. Fibroblast growth factor 23 (FGF23) is an important hormone involved in the regulation of serum phosphate levels. Whether FGF23 contributes to hypophosphatemia due to drug-induced FS is unknown. An association between FS and FGF23 has been reported, but the findings have been inconsistent. ${ }^{7-11}$ Pathogenesis of drug-induced FS may involve mitochondrial damage in the proximal tubules. ${ }^{12}$

\footnotetext{
Abbreviations:

CKD, chronic kidney disease; COI, cutoff index; FGF23, fibroblast growth factor 23; FS, Fanconi syndrome; GFR, glomerular filtration rate; HBsAg, surface antigen of hepatitis B virus; HBV DNA, hepatitis B virus DNA; HIV, human immunodeficiency virus; MDRD, Modification of Diet in Renal Disease; TmPi/GFR, ratio of the renal tubular maximum reabsorption rate of phosphate to the glomerular filtration rate
}

\section{Corresponding author : Eun Young Lee}

Department of Internal Medicine, Soonchunhyang University Cheonan Hospital, 31 Soonchunhyang 6-gil, Cheonan 31151, Korea

Tel: +82-41-570-3684, Fax: +82-41-574-5762

E-mail: eylee@sch.ac.kr

http://orcid.org/0000-0002-4513-9888

Received : Feb. 20, 2017/ Revised : May 11, 2017 / Accepted : Jun. 7, 2017

Copyright ( 92018 by Korean Association for the Study of the Liver

This is an Open Access article distributed under the terms of the Creative Commons Attribution Non-Commercial License (http://creativecommons.org/licenses/by-nc/3.0/) which permits unrestricted non-commercial use, distribution, and reproduction in any medium, provided the original work is properly cited. 
We evaluated a woman with chronic hepatitis $B$ receiving adefovir who presented with proteinuria, hypophosphatemia, progressive bone pain throughout the body, and bone fractures. Eventually, she was diagnosed with FS associated with adefovir. FGF23 levels and mitochondria in proximal tubules were evaluated. Here, we report the FGF23 levels and results of renal biopsy in the case of adefovir-induced hypophosphatemic osteomalacia. Our data suggest that mitochondrial damage in the proximal tubules is critical in the pathogenesis of drug-induced FS.

\section{CASE REPORT}

A 36-year-old woman visited the nephrology department of Soonchunhyang University Cheonan Hospital with progressive multiple bone pain and proteinuria. She was not independently mobile and was using a wheelchair. She had a 9- and 7-year history of $100 \mathrm{mg} /$ day lamivudine and $10 \mathrm{mg} /$ day adefovir, respectively, for chronic hepatitis $B$. Three years previously, she received surgery for a non-traumatic right femur neck fracture. A ${ }^{99 \mathrm{~m}} \mathrm{Tc}$ bone scan was performed due to muscle weakness and multiple bone pain. Multiple hot bone uptakes were noted in the right clavicle, both ribs, and pelvis (Fig. 1A). Dual-energy X-ray absorp- tiometry revealed decreased lumbar spine bone mineral density of $0.786 \mathrm{~g} / \mathrm{cm}^{2}$ (T-score, -2.8 ; Z-score, -2.5 ).

On admission, multiple bone pain with tenderness was noted on physical examination, and vital signs were unremarkable. The patient's weight and body mass index were $47.4 \mathrm{~kg}$ and $18.9 \mathrm{~kg} / \mathrm{m}^{2}$, respectively. She denied a history of hypertension and diabetes, and the use of any other medications. The results of serology markers for quantitative surface antigen of hepatitis B virus (HB$\mathrm{sAg}$ ) was 6,481 cutoff index (COI) with positive hepatitis $\mathrm{E}$ antigen, negative hepatitis $E$ antibody, and undetectable hepatitis virus B DNA (HBV DNA). Laboratory analyses revealed white blood cell count, 4,540/ $\mu \mathrm{L}$; hemoglobin, $13.8 \mathrm{~g} / \mathrm{dL}$; hematocrit, 41.2\%; platelet count, 289,000/ $\mu \mathrm{L}$; total bilirubin, $0.3 \mathrm{mg} / \mathrm{dL}$; aspartate aminotransferase, $17 \mathrm{IU} / \mathrm{L}$; alanine aminotransferase, $12 \mathrm{IU} / \mathrm{L}$; lactate dehydrogenase, $150 \mathrm{IU} / \mathrm{L}$; high-sensitivity C-reactive protein, $1.22 \mathrm{mg} / \mathrm{L}$; prothrombin time, 12.1 seconds; activated partial thromboplastin time, 37.8 seconds; serum osmolality, $287 \mathrm{mOsm} / \mathrm{kg}$; and osmolar gap, $2 \mathrm{mOsm} / \mathrm{kg}$. Serum creatinine level was within normal limits and marked hypophosphatemia and hypouricemia were noted (Table 1). The elevated alkaline phosphatase implied the presence of metabolic bone disease, but intact parathyroid hormone and vitamin D levels were noted within normal limits. Arterial-blood gas analysis values were as follows: $\mathrm{pH}, 7.386$; $\mathrm{PaCO}_{2}$,

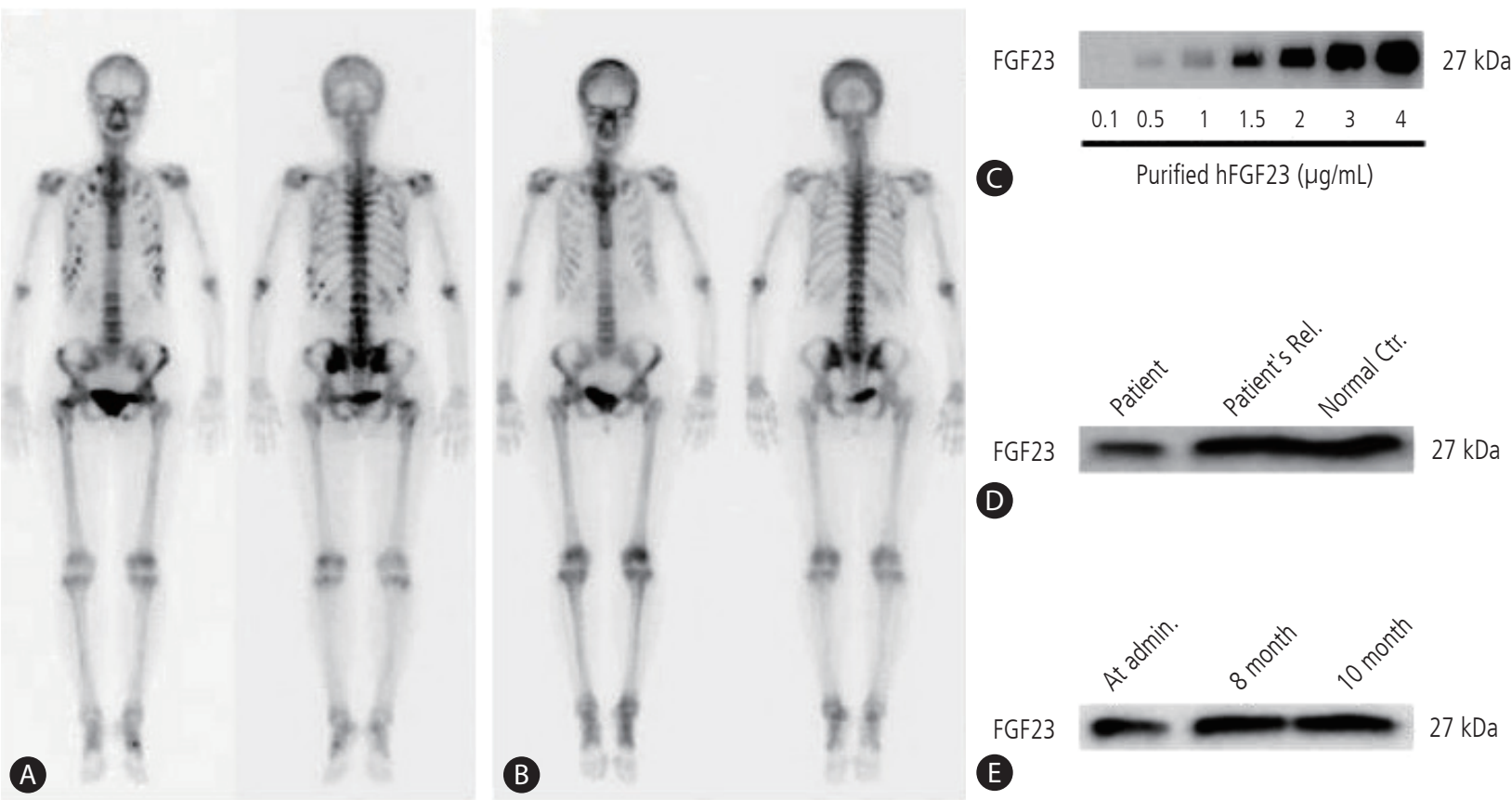

Figure 1. ${ }^{99 m} \mathrm{Tc}$-bone scan and serum FGF23 level in patients. (A, B) ${ }^{99 \mathrm{~m}} \mathrm{Tc}$-bone scan showing multiple hot spots of bone uptake in the right clavicle, both ribs, and pelvis before (A) and after (B) 8 months of treatment. (C) Validation of FGF23 antibody levels using immunoblotting. Purified human FGF23 (hFGF23) was detected by Western blotting. (D) Serum FGF23 levels of the patient, the patient's sister (Patient's Rel.), and an unrelated normal subject (Normal Ctr.) at admission. (E) Serum FGF23 levels at administration (Admin.) and after treatment (8 and 10 months). Rel., relative; Ctr., control. 
Samel Park, et al.

Adefovir-induced Fanconi syndrome

Table 1. Biochemical data at admission and 8 months after adefovir withdrawal

\begin{tabular}{|c|c|c|c|}
\hline & Initial & 8 months & Reference range \\
\hline \multicolumn{4}{|l|}{ Serum } \\
\hline Total protein (g/dL) & 7.1 & 6.8 & $6.7-8.6$ \\
\hline Albumin (g/dL) & 4.6 & 4.3 & $3.5-5.5$ \\
\hline Glucose (mg/dL) & 86 & 90 & $75-100$ \\
\hline BUN (mg/dL) & 12.0 & 11.0 & $7.0-20.0$ \\
\hline Creatinine (mg/dL) & 0.9 & 0.8 & $0.5-1.2$ \\
\hline Sodium (mmol/L) & 138 & 139 & $136-145$ \\
\hline Potassium (mmol/L) & 3.8 & 3.7 & $3.5-5.0$ \\
\hline Chloride (mmol/L) & 105 & 106 & $102-109$ \\
\hline Bicarbonate $(\mathrm{mmol} / \mathrm{L})$ & 19.0 & 23.5 & $22-30$ \\
\hline Calcium (mg/dL) & 9.0 & 9.0 & $8.7-10.2$ \\
\hline Phosphate (mg/dL) & 1.5 & 3.3 & $2.5-4.3$ \\
\hline Uric acid (md/dL) & 1.5 & 2.8 & $2.5-5.6$ \\
\hline Alkaline phosphatase (IU/L) & 343 & 198 & $33-96$ \\
\hline Intact PTH (pg/mL) & 27.68 & & $8.0-51.0$ \\
\hline $25(\mathrm{OH})$ vitamin D (ng/mL) & 22.2 & & $20-50$ \\
\hline 1,25(OH)2 Vitamin D3 (pg/mL) & 33.84 & & $15-75$ \\
\hline \multicolumn{4}{|l|}{ Urine } \\
\hline Glucose & $2+$ & Negative & Negative \\
\hline Glucose (mg/day) & 1,839 & 3.9 & $50-300$ \\
\hline Protein & Trace & Negative & Negative \\
\hline Protein (mg/day) & 465.3 & 95.6 & $<150$ \\
\hline Sodium (mmol/day) & 131.4 & 119.4 & $100-260$ \\
\hline Potassium (mmol/day) & 53.5 & 32.9 & $25-100$ \\
\hline Phosphate (mg/day) & 894.6 & 649.4 & $400-1300$ \\
\hline Calcium (mg/day) & 529.4 & 406.3 & $<300$ \\
\hline Uric acid (mg/day) & 707.4 & 562.7 & $250-800$ \\
\hline Creatinine (mg/day) & $1,002.6$ & 1052.5 & $1000-1600$ \\
\hline B2-MG/Cr (mg/g Cr) & $27,532.6$ & & \\
\hline FEUA (\%) & 42.4 & 20.9 & \\
\hline FEPi (\%) & 53.5 & 17.6 & \\
\hline TRPi (\%) & 46.5 & 82.4 & \\
\hline TmPi/GFR (mg/dL) & 0.70 & 2.31 & \\
\hline
\end{tabular}

BUN, blood urea nitrogen; PTH, parathyroid hormone; B2-MG/Cr, B2-microglobulin/creatinine; FEUA, fractional excretion of uric acid; FEPi, fractional excretion of phosphate; TRPi, fractional tubular reabsorption of phosphate; TmPi/GFR, ratio of the renal tubular maximum reabsorption rate of phosphate to the glomerular filtration rate.

$35.7 \mathrm{mmHg} ; \mathrm{PaO}_{2} 110.4 \mathrm{mmHg} ; \mathrm{HCO}_{3}$; $20.9 \mathrm{mEq} / \mathrm{L}$; base excess, -3.5 mEq/L; $\mathrm{O}_{2}$ saturation, 98.0\%; and anion gap, $12.1 \mathrm{mEq} / \mathrm{L}$. Urinalysis with microscopic examination showed $\mathrm{pH}, 7.0$; specific gravity of 1.012; trace protein; two positives of glucose; 5 to 9 red blood cells per high-power field; and 1 to 4 white blood cells per high-power field. Twenty four-hour urine analysis revealed generalized amino-aciduria and impaired reabsorption of other solutes, indicating proximal tubule dysfunction (Table 1). Blood aluminum levels and 24-hour urine aluminum levels were $2.38 \mu \mathrm{g} / \mathrm{L}$ and 2.2 $\mu \mathrm{g} /$ day, respectively. Antinuclear antibody, antibody to double- 


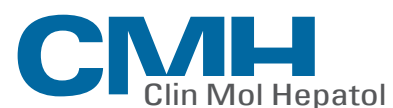

Volume 24 Number_3 September 2018

stranded DNA, and anti-neutrophil cytoplasmic antibody were all negative. Hormone levels measured at 8:00 AM revealed the following: adrenocorticotropic hormone, $9.71 \mathrm{pg} / \mathrm{mL}$; cortisol, 8.13 $\mu \mathrm{g} / \mathrm{dL}$; luteinizing hormone, $3.39 \mathrm{mIU} / \mathrm{mL}$; follicle stimulating hormone, $5.53 \mathrm{mIU} / \mathrm{mL}$; and estradiol $53.23 \mathrm{pg} / \mathrm{mL}$. There was no evidence of dysproteinemia. Renal biopsy confirmed normal renal pathology in light microscopy, and immunofluorescence stains were negative. In electron microscopy, the glomerulus appeared normal but there was mitochondrial damage in proximal tubules; structures in distal tubules were near normal (Fig. 2). Finally, we made a diagnosis of FS. To measure serum FGF23 levels, immunoblotting using a standard curve with FGF23 validation of purified human FGF23 antibody (Fig. 1C) was performed..$^{13}$ Serum FGF23 levels were lower than those of normal controls (Fig. 1D).
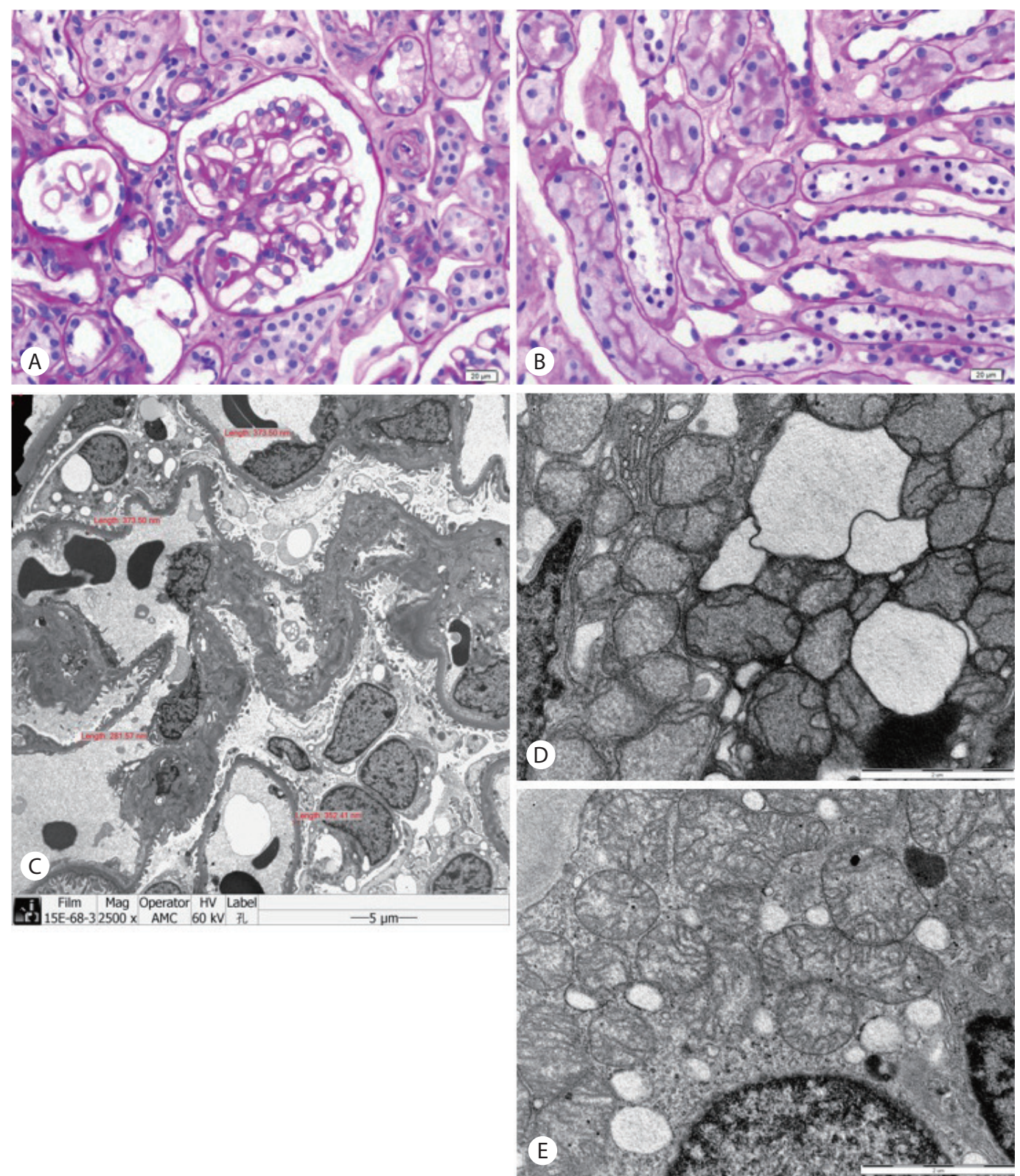

Figure 2. Renal pathology of the patient. The glomerulus (A) and tubules (B) were normal in light microscopy (Periodic acid-Schiff stain, the bar represents $20 \mu \mathrm{m}$ ). (C) There were nonspecific findings in conventional electron microscopy performed to detect the presence of electron-dense deposits or microstructures in patients with suspicion of glomerulonephritis (the bar represents $5 \mu \mathrm{m}$ ). (D) Severe mitochondrial damage of proximal tubules was revealed in focused electron microscopy. (E) The structures of mitochondria in distal tubules had a near-normal appearance (in picture D and E, the bar represent $2 \mu \mathrm{m}$ ) 
Adefovir and lamivudine were replaced with $1 \mathrm{mg} /$ day of entecavir, and phosphate (2,000 mg/day of K-phos original tablet containing $114 \mathrm{mg}$ of phosphorus and $144 \mathrm{mg}$ of potassium in a 500 $\mathrm{mg}$ tablet) and $0.25 \mu \mathrm{g} /$ day of calcitriol were added. After 2 months of treatment, the level of serum phosphate was markedly increased (3.4 mg/dL). The patient's bone pain was markedly improved and she was able to perform normal daily activities and could walk again without a wheelchair. After 8 months of treatment, the ratio of the renal tubular maximum reabsorption rate of phosphate to the glomerular filtration rate (TmPi/GFR) was increased to $2.31 \mathrm{mg} / \mathrm{dL}$, and other laboratory markers indicating proximal tubule dysfunction were normalized (Table 1). Multiple hot uptakes apparent in the first ${ }^{99 \mathrm{~m}} \mathrm{Tc}$-bone scan had disappeared (Fig. 1B) and serum FGF23 level had normalized (Fig. 1E). The quantitative HBsAg was 5,292 COI without hepatitis B DNA detection, and the status of hepatitis $E$ antigen and antibody was not changed after switch to entecavir.

\section{DISCUSSION}

We report a case with mitochondrial damage in the proximal tubules and decrease of the FGF23 levels in a patient with adefovir-induced hypophosphatemic osteomalacia and chronic hepatitis B infection. FS can present as hypophosphatemic osteomalacia, which is attributed to the impaired reabsorbing phosphate in the proximal renal tubule. ${ }^{6}$ Nephrotoxicity associated with high dose of adefovir, $120 \mathrm{mg} /$ day, used in patients with HIV is well known.' Adefovir has an adverse event profile similar to that of a placebo when used at a dose of $10 \mathrm{mg} /$ day in patients with chronic hepatitis B infections. ${ }^{2}$ However, nephrotoxicity including FS and hypophosphatemic osteomalacia has been described in several reports in patients taking a low dose of $10 \mathrm{mg} /$ day. ${ }^{3,4}$

Presently, the patient's symptoms were nonspecific. Symptoms including myalgia, general weakness, or multiple fracture without an explainable cause have been described. ${ }^{3,4}$ In our opinion, suspicion of FS is most important to make a diagnosis, and urinalysis is the most valuable screening test for FS. FS should be considered in non-diabetic patients when chronic glycosuria is evident and when a tubular range of proteinuria exists or when the levels of phosphorus and uric acid are lower than normal range or in the lower normal margin. The temporal relationship between exposure to medications with known proximal tubule toxicity and the development of tubulopathy ${ }^{12}$ suggests the possibility of FS. In such patients, diagnosis as FS is aided by evaluation for excretion of phosphorus or uric acid, such as fractional excretion of uric acid, fractional excretion of phosphate, fractional tubular reabsorption of phosphate, and TmPi/GFR. ${ }^{12}$

FGF23 is a principle hormone regulating renal phosphate handling, and produced by osteocytes and osteoblasts in bone. In chronic kidney disease (CKD), FGF23 is increased, which enhance the excretion of phosphate and in turn, restores normophosphatemia. ${ }^{14}$ In contrast, FGF23 levels are decreased in hypophosphatemic patients with vitamin D deficiency, FS, and Cushing's syndrome by an ectopic ACTH-producing tumor. ${ }^{7}$ The present study showed that serum FGF23 level was markedly decreased in this patient with severe hypophosphatemia prior to treatment and normalized after treatment. The findings from previous study are inconsistent. Whereas normal levels of FGF23 were reported in patients treated with tenofovir in one study, ${ }^{8}$ another study described elevated FGF23 in tenofovir-related hypophosphatemia, ${ }^{9}$ and with adefovir treatment. ${ }^{10}$ Yet, a recent study by Goto $\mathrm{S}$, et al. described low FGF23 levels in 2 patients with adefovir-induced FS. ${ }^{11}$ Among 5 patients in the previous study above, 2 patients received tenofovir due to infection with HIV, and the other patients received adefovir due to chronic hepatitis $B$ infection. The collective data suggest that FGF23 levels may not be strictly associated with either tenofovir or adefovir, or the infecting virus. We suggest that FGF23 does not influence the phosphorus level in secondary FS.

FGF23 could be affected by many contributing factors. These include renal function ${ }^{15}$ and the amount of dietary phosphorus. ${ }^{16}$ The most important reason for the conflicting results to date is probably the differing glomerular filtration rate (GFR), since GFR is a major determinant of FGF23 levels. ${ }^{15}$ In previously reported cases, all the patients with secondary FS displayed mild to moderate renal impairment, in which GFR were approximately from 50 to $70 \mathrm{~mL} / \mathrm{min} / 1.73 \mathrm{~m}^{2}$, referred to CKD stage between 2 to $3 .^{17}$ In one case involving a 75-year-old woman, estimated GFR using the Modification of Diet in Renal Disease (MDRD) formula was 72.9 $\mathrm{mL} / \mathrm{min}^{10}{ }^{10}$ Thus, there is a possibility that estimated GFR using the MDRD formula could overestimate renal function, especially in more elderly woman. Conversely, our patient was young adult with normal renal function and did not have any other cause, such as hypertension, diabetes, or history of any other medications, except adefovir.

The renal biopsy revealed mitochondrial damage in the proximal tubules and no abnormalities in the glomerulus and distal tubules. After cessation of adefovir, the impaired solute reabsorption in the proximal tubules and the symptoms suggestive of osteomala- 
cia disappeared. Thus, we suggest that mitochondrial damage in the proximal tubules is critical in drug-induced FS. In addition, these results suggest that FGF23 does not influence the occurrence of hypophosphatemia in drug-induced FS, and is independently influenced by factors including blood phosphorus level, amount of phosphorus intake, and GFR in FS.

In conclusion, adefovir induces proximal tubular mitochondrial injury and subsequent proximal tubular phosphate wasting, which results in hypophosphatemia and osteomalacia. The decreased level of FGF23 is a consequence, not a cause, of hypophosphatemia.

\section{Authors' contribution}

Park S.E. and Lee E.Y. have a responsibility as the first author and the corresponding author, respectively. Kim W.I. and Cho D.H. acquired the clinical data. Kim Y.J. and Kim H.S. helped to diagnose Fanconi syndrome and to exclude other possible diseases. Kim J.H. and Cha S.K. evaluated and interpreted the level of FGF23. Park K.S. and Lee J.H. analyzed the result of light microscopy and electron microscopy. Lee S.M. adviced to lead a diagnosis of Fanconi syndrome by interpreting bone scan.

\section{Financial support}

This research was supported by Basic Science Research Program through the National Research Foundation of Korea (NRF) funded by the Ministry of Education (2015R1A6A1A03032522, 2017R1D1A3B03027898), Korea Health Technology R\&D Project through the Korea Health Industry Development Institute (KHIDI) funded by the Ministry of Health \& Welfare (HI17C-2059-010017), and Soonchunhyang University Research Fund.

\section{Conflicts of Interest}

The authors have no conflicts to disclose.

\section{REFERENCES}

1. Kahn J, Lagakos S, Wulfsohn M, Cherng D, Miller M, Cherrington J, et al. Efficacy and safety of adefovir dipivoxil with antiretroviral therapy: a randomized controlled trial. JAMA 1999;282:2305-2312.

2. Hadziyannis SJ, Tassopoulos NC, Heathcote EJ, Chang TT, Kitis G, Rizzetto $\mathrm{M}$, et al. Adefovir dipivoxil for the treatment of hepatitis $B$ e antigen-negative chronic hepatitis B. N Engl J Med 2003;348:800-807.

3. Jung YK, Yeon JE, Choi JH, Kim CH, Jung ES, Kim JH, et al. Fanconi's syndrome associated with prolonged adefovir dipivoxil therapy in a hepatitis B virus patient. Gut Liver 2010;4:389-393.

4. Girgis CM, Wong T, Ngu MC, Emmett L, Archer KA, Chen RC, et al. Hypophosphataemic osteomalacia in patients on adefovir dipivoxil. J Clin gastroenterol 2011;45:468-473.

5. Laing CM, Toye AM, Capasso G, Unwin RJ. Renal tubular acidosis: developments in our understanding of the molecular basis. Int I Biochem Cell Biol 2005;37:1151-1161.

6. Earle KE, Seneviratne T, Shaker J, Shoback D. Fanconi's syndrome in HIV+ adults: report of three cases and literature review. J Bone Miner Res 2004;19:714-721.

7. Endo I, Fukumoto S, Ozono K, Namba N, Tanaka H, Inoue D, et al. Clinical usefulness of measurement of fibroblast growth factor 23 (FGF23) in hypophosphatemic patients: proposal of diagnostic criteria using FGF23 measurement. Bone 2008;42:1235-1239.

8. Saidenberg-Kermanac'h N, Souabni L, Prendki V, Prie D, Boissier MC. Normal plasma FGF23 levels kinetic in tenofovir-related hypophosphatemic osteomalacia in an HIV-infected patient with von Recklinghausen disease. Joint Bone Spine 2011;78:306-308.

9. Saeedi R, Jiang SY, Holmes DT, Kendler DL. Fibroblast growth factor 23 is elevated in tenofovir-related hypophosphatemia. Calcif Tissue Int 2014;94:665-668.

10. Ichchou L, Rist S, Lespessailles E, Prie D, Benhamou CL. Reversible increase in FGF23 in a hypophosphatemic renal and bone disease linked to antiviral therapy by adefovir. Joint Bone Spine 2013;80:668-669.

11. Goto S, Fujii H, Kono K, Watanabe K, Nakai K, Nishi S. Serum FGF23 levels may not be associated with serum phosphate and 1,25-dihydroxyvitamin D levels in patients with Fanconi syndrome-induced hypophosphatemia. Clin Kidney J 2016;9:677-681.

12. Hall AM, Bass P, Unwin RJ. Drug-induced renal Fanconi syndrome. QJM 2014;107:261-269

13. Taylor SC, Berkelman T, Yadav G, Hammond M. A defined methodology for reliable quantification of Western blot data. Mol Biotechnol 2013;55:217-226

14. Juppner H. Phosphate and FGF-23. Kidney Int Suppl 2011;79121:S24S27.

15. Filler G, Liu D, Huang SH, Casier S, Chau LA, Madrenas J. Impaired GFR is the most important determinant for FGF-23 increase in chronic kidney disease. Clin Biochem 2011;44:435-437.

16. Goto S, Nakai K, Kono K, Yonekura Y, Ito J, Fujii H, et al. Dietary phosphorus restriction by a standard low-protein diet decreased serum fibroblast growth factor 23 levels in patients with early and advanced stage chronic kidney disease. Clin Exp Nephrol 2014;18:925-931.

17. Kidney Disease: Improving Global Outcomes CKD Work Group. KDIGO 2012 clinical practice guideline for the evaluation and management of chronic kidney disease. Kidney Int 2013;3:1-150. 